\title{
An analysis of light pollution at the Thirty Meter Telescope candidate sites
}

Reed L. Riddle, David Walker, Matthias Schöck, Sebastian G. Els, Warren Skidmore, et al.

Reed L. Riddle, David Walker, Matthias Schöck, Sebastian G. Els, Warren Skidmore, Tony Travouillon, Edison Bustos, Juan Seguel, Joselino Vasquez, Robert D. Blum, Paul Gillett, Brooke Gregory, "An analysis of light pollution at the Thirty Meter Telescope candidate sites," Proc. SPIE 7012, Ground-based and Airborne Telescopes II, 701223 (10 July 2008); doi: $10.1117 / 12.787295$

Event: SPIE Astronomical Telescopes + Instrumentation, 2008, Marseille, France 


\title{
An analysis of light pollution at the Thirty Meter Telescope candidate sites
}

\author{
Reed L. Riddle ${ }^{a}$, David Walker ${ }^{b}$, Matthias Schöck $^{a}$, Sebastian G. Els ${ }^{b}$, Warren Skidmore ${ }^{a}$, \\ Tony Travouillon ${ }^{a}$, Edison Bustos ${ }^{b}$, Juan Seguel $^{b}$, Joselino Vasquez ${ }^{b}$, Robert D. Blum ${ }^{c}$, Paul \\ Gillett $^{a}$, Brooke Gregory ${ }^{b}$ \\ ${ }^{a}$ TMT Observatory Corporation, 2632 E. Washington Blvd., Pasadena, CA, 91107 USA \\ ${ }^{b}$ Cerro Tololo Inter-American Observatory, Casilla 603, La Serena, Chile \\ ${ }^{c}$ National Optical Astronomy Observatory, 950 N. Cherry Ave., Tucson, 85719 USA
}

\begin{abstract}
Light pollution can create difficulties for astronomers attempting to observe faint objects in the night sky. Light from a local small town can be just as intrusive as light from a large city in the distance. As the population of the Earth increases, light pollution will become more of a problem, even in remote areas. The Thirty Meter Telescope site testing program has measured light pollution at the candidate sites by using all sky cameras; an analysis procedure enhances the all sky camera images to make the determination of the effects of the light pollution. This paper summarizes the light pollution analysis procedure and current results, which are that light pollution is currently unimportant for TMT to select a site for the final telescope location.
\end{abstract}

Keywords: Instruments, all sky cameras; site testing; light pollution

\section{INTRODUCTION}

The Thirty Meter Telescope project (TMT) is examining five sites in Chile, Hawaii and Mexico as possible locations for the placement of the telescope. As part of the site testing program, images are taken of the night sky using the All-Sky Camera (ASCA). This camera is designed to image clouds in the sky for determination of the statistics of cloud measurements and usable observing nights at each site.

There is additional information about the night sky that would be useful in the decision on where to place TMT. For any telescope, the amount of ambient light scattered into the optical path can degrade the observational capability of the instrument. It would be a waste to build a large light collecting aperture and then place it in a location where the sky is too bright to take advantage of the full capability of the telescope.

A procedure was developed to extract light pollution information from the ASCA data set. The ASCA imaging system was not designed to observe light pollution, so some thought and care went into developing a method to examine the light pollution at the sites. This method was designed only to determine if light pollution would be a factor in the selection of any of the TMT candidate sites. More extensive work is underway to quantify the results and to extract more information (such as temporal variability of the light pollution sources) from the data if possible.

\section{LIGHT POLLUTION AND ASTRONOMY}

Historically, the performance of a telescope has been limited by the quality of the site where it has been constructed. While adaptive optics systems have started to free telescopes from the limits of atmospheric turbulence, scattered light in the atmosphere remains a limiting factor for ground based optical telescopes. Placing a telescope inside of a city immediately limits the magnitude of the faintest stars that it can observe. With a telescope as large as TMT, a dark site, as far away from any scattered light of human civilization as possible, is the only

\footnotetext{
Author contact information: Send correspondence to Reed Riddle. E-mail: riddle@tmt.org, Telephone: 1626395 8956
}

Ground-based and Airborne Telescopes II, edited by Larry M. Stepp, Roberto Gilmozzi, Proc. of SPIE Vol. 7012, 701223, (2008) · 0277-786X/08/\$18 - doi: 10.1117/12.787295 
way to guarantee that the observations will take full advantage of the sensitivity of such a large light collecting area.

In the early part of the twentieth century, when the first large reflecting telescopes were being constructed, light pollution was not a consideration when deciding where to site the telescopes. There was so little lighting that factors such as accessibility, clear nights and good weather were far more important, and the growth of modern America and its lighting was not foreseen. That is why many observatories (Mt. Wilson, Palomar, Kitt Peak) were constructed where they are; at the time of construction all of those sites were dark sites. As time progressed, those sites were more and more affected by city lights. ${ }^{1,2}$

Later in the twentieth century, it was realized that light pollution was a growing threat to astronomy, especially in the southwestern United States. ${ }^{3}$ Projects were undertaken to measure light pollution and its effects on observatories, ${ }^{4,5}$ and understand how it affected the quality of observations. ${ }^{6}$ Perhaps the most effective movement in controlling light pollution has been educating the public, both about how light pollution affects astronomy ${ }^{7,8}$ and how it also wastes energy resources and affects other aspects of lives of ordinary people..$^{9,10}$

Protecting sites and improving the light pollution situation in already developed sites has become an important aspect of ground based astronomy in recent years. ${ }^{11,12}$ There have been some notable successes in convincing cities to limit light pollution through laws or other actions. ${ }^{13}$ This has expanded to astronomical sites outside of the United States; projects to monitor the change in light pollution ${ }^{14}$ and actions of governments in promoting the control of light pollution ${ }^{15}$ continue.

\section{A SHORT DESCRIPTION OF THE ALL SKY CAMERA}

The All-Sky Camera (ASCA) is a replica of the unit mounted at CTIO, and is used in both the TMT and LSST site testing campaigns. ${ }^{16}$ ASCA consists of a small cylinder with a clear plexiglas half dome sealed to the top. Inside, there is a large fisheye lens, the CCD, and various fans and power supplies. There is also a filter wheel, and a Stealth Computer Corporation ${ }^{17}$ computer inside to control the system. The software is a custom suite of applications designed to obtain the necessary data.

ASCA is a self contained unit; there is little interaction with the TMT site testing system as ASCA is a simpler instrument. ASCA has a dedicated server, located in La Serena, Chile at the CTIO headquarters, which takes the images from the CCD and downloads them directly throughout the night. The images are downloaded to the server as they are taken on a five minute cycle; the images are processed by the server and displayed on a website. Since the site testing telescope can be seen in the images, ASCA is also used as a check on the telescope status if there are problems during the night.

ASCA takes an image of the entire sky once every five minutes through the night in four bands (b,r, y, z), and images every three hours using an Na filter. The images are used to determine the large scale conditions of the night sky. The methods used to examine cloud fraction and observable time for the TMT site testing project are discussed elsewhere in this proceeding. ${ }^{18}$

The only instrument in the TMT site testing suite that could possibly measure light pollution is ASCA. With its observations of the sky to the horizon, it is possible to see the effects of light pollution, even in single images. However, although ASCA is designed to view the sky to the horizon, it is not optimized to do so. The fisheye lens compresses the horizon to a smaller fraction of the total CCD area compared to observations around the zenith. This is perfect for measuring cloud fractions in the TMT observing window (zenith angles above 65 degrees), but not for measurements on the horizon. Since light pollution is expected near the horizon, this complicates measuring any effects from it using ASCA.

In addition to the limited fraction of the CCD dedicated to the horizon, there is also a limitation on using a single image to measure light pollution. While light from a bright source can show up in individual images, it is very faint compared to the other features in the frame. This makes it difficult to determine the angular coverage of the scattered light. The Na filter could measure light pollution, but the images from this usually do not clearly show the light from nearby cities (possibly because they have few Na light systems). Longer exposure times may help with the problem, but that would interfere with the main function of ASCA in measuring the cloud fractions (which is a more important limitation on the site decision for TMT than light pollution). Therefore, a method had to be developed that uses the gathered data to determine information about light pollution at the sites. 


\section{THE METHOD OF ANALYZING ASCA IMAGES FOR LIGHT POLLUTION}

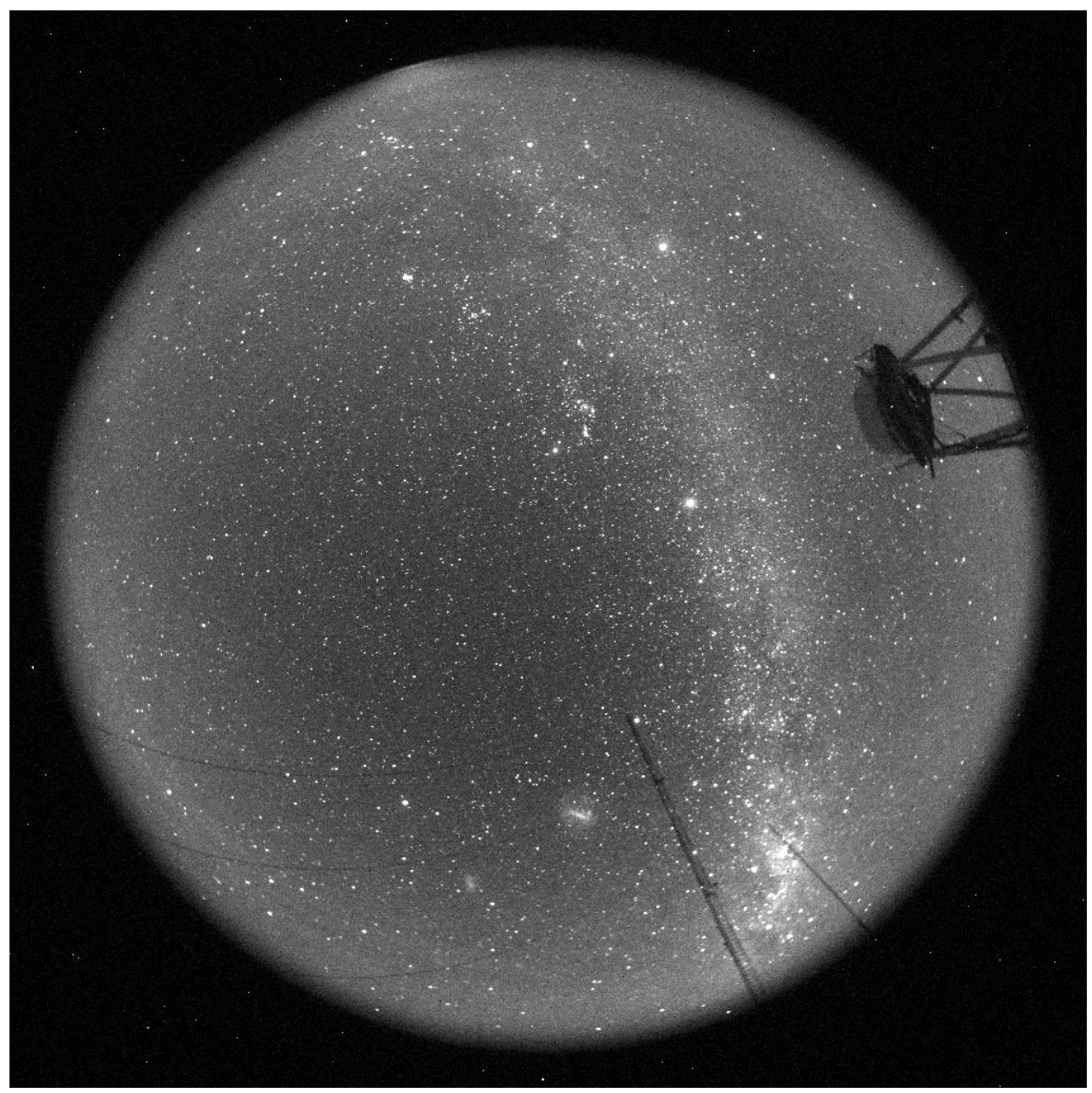

Figure 1. A single ASCA image from Cerro Armazones taken on the night of December 8, 2007.

Figure 1 is a raw ASCA images in the $\mathrm{b}$ filter observed from the TMT site testing station at Cerro Armazones in Chile. As with all other ASCA images presented in this paper, north is to the top, and east to the right. This particular image is corrected for dark current. Dark current correction was not originally included in the ASCA observing program, so some images are dark corrected and some show dark current effects (hot pixels in the dark corners are the clearest indication). There is no flat fielding or bias correction for the ASCA images.

This particular image was taken on a clear, new Moon night, at a time deep in astronomical twilight when any city lights should still be prevalent (i.e. early enough for people to still be out). The image is dominated by stars and the Milky Way, with the Large and Small Magellanic Clouds, the constellation Orion and several other features easily identifiable. The site testing telescope is to the right in the image, and the ground layer measurement tower at the bottom.

The city of Antofagasta is just to the west of north in this image, at the top at about $345^{\circ}$. Note that the light pollution is not very noticeable on the horizon in this image; it nearly lines up with the Milky Way, and could almost be mistaken for the galaxy if it was a bit fainter. This illustrates the difficulty of determining light pollution from a single image, even using the $\mathrm{b}$ filter (which should show scattered light the most clearly). A single image does not enhance the light pollution compared to the star and galaxy light, and faint sources of light pollution (unlike Antofagasta, a city of 300,000 people) may not appear in a single image at all. 


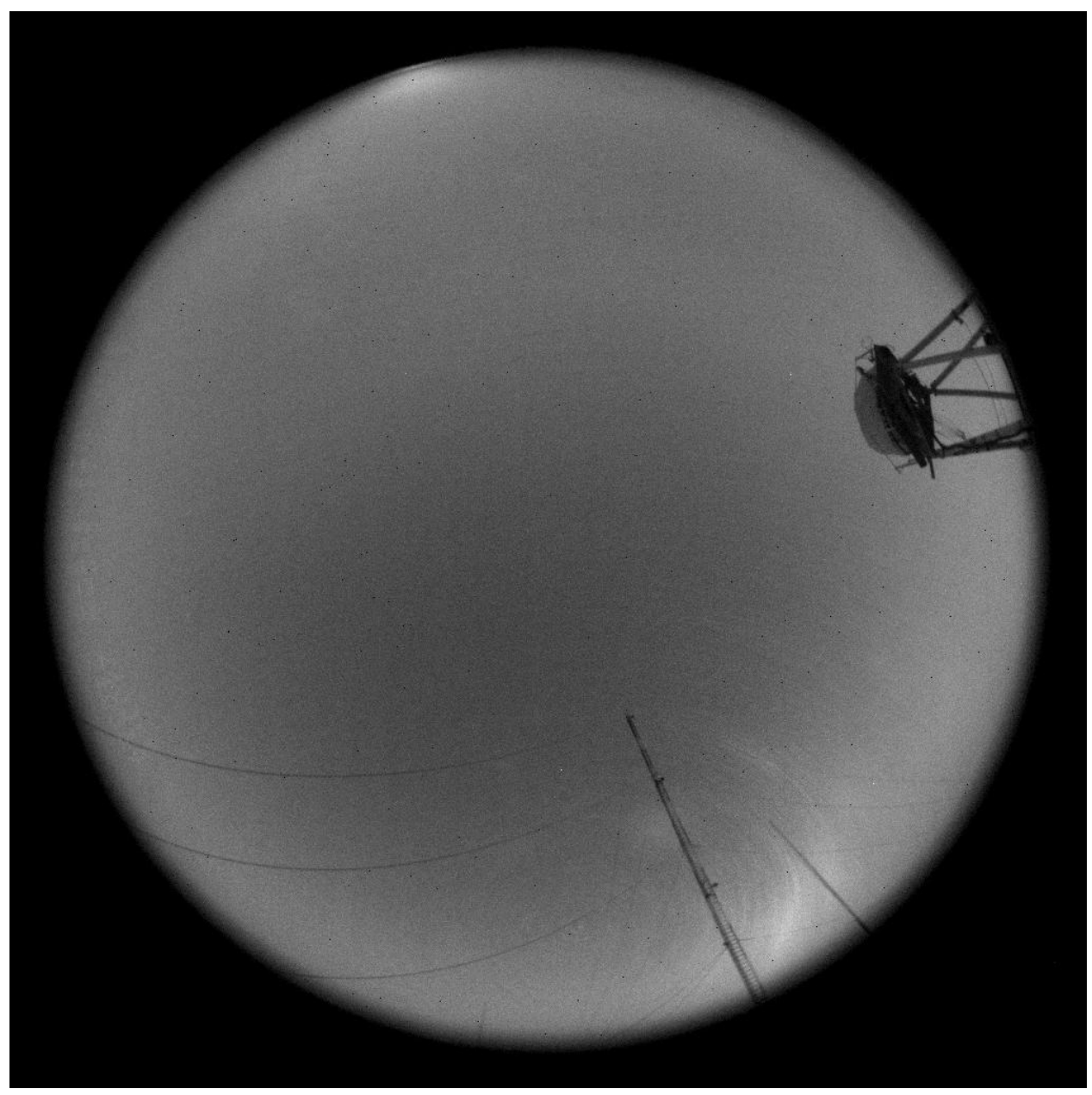

Figure 2. The $10 \%$ median image for the same night as the image shown in Figure 1.

With the difficulty in measuring light pollution from single ASCA images apparent, another way to tease out the effects from light pollution was required. The ASCA system was not designed to measure light pollution or image the horizon clearly, which makes extracting light pollution information a challenge. The final method had to enhance the light pollution effects in the image while removing or minimizing the brighter sources in the image. In addition, the method had to measure the light pollution at the horizon without contaminating it with astronomical sources such as starlight, Sun or Moon light, or light from the galactic plane.

Clear nights at new Moon were selected for the light pollution data set. This selection gives the longest stretch of images to use in the analysis with images free of effects from the bright light of the Moon. Images in the late evening and early morning that contained effects from sunset were removed from the data set. Several tests were then made on the remaining images to develop a method for light pollution measurement. Most of the tests failed to enhance the light pollution at the edge of the ASCA image, or they increased the brightness of the astronomical objects and contaminated the light pollution measurements.

One method did show promise. Through the night, the stars and galaxy move from pixel to pixel, but at times the pixels do not have any astronomical objects in them. A median image was created that takes the values from each pixel across the night and finds the median. That creates an image that is a median of the ASCA observations during the night per pixel (each pixel is a median of what the ASCA measured independently in that pixel through the night). At the median level, there still are effects from stars in some pixels, and the effect of the galaxy can be quite large. However, the light pollution scattering on the horizon is well defined and clearly 
evident; this method achieves the goal of enhancing the light pollution while removing some of the effects of the astronomical objects.

Figure 2 is one of these images; it is for the same site and night as Figure 1. The final "median method" uses the pixel values at the $10 \%$ darkest pixel level; this was found to be the best compromise between dark sky and avoiding low signal pixel issues. In the figure, there is no evidence of single stars; some pixels show as dark or hot, but those are due to dark current correction and not physical effects. The galaxy is apparent as a small smear near the tower, and some smearing from stars rotating around the pole is evident; objects that do not move across the frame rapidly through the night show up at the $10 \%$ level as expected.

The reason this method was chosen is clearly apparent in the difference between the appearance of the scattered light from Antofagasta between Figures 1 and 2. In the 10\% median image, the scattered light is a strong source on the horizon, and reaches into the frame; in the single image, it is difficult to see the scattered light without realizing it is there and examining the image closely. This enhancement makes it a much simpler process to find light sources on the horizon, especially faint ones that do not appear at all in a single ASCA image.

\subsection{Features of the Light Pollution Analysis}

Figure 2 is a fairly clean light pollution analysis image; there are few features that are not due to light pollution, and the light pollution is clear in the frame. Unsurprisingly, not all of the images are quite as simple to interpret.

Figure 3 is a set of light pollution analysis images, at the same $10 \%$ median level, from the ASCA system on Cerro Tolar. These images show many of the features that can appear in the ASCA data and how they affect the analysis. First, note that all of the images are not corrected for dark current; this increases the noise in the image, but not to the point that the analysis cannot be done. Also, note that the level of the sky in the images is not the same, and it can vary across the sky as well. This is not a real effect; it is likely due to the small differences in CCD sensitivity and how the brightness in the sky may have varied through the night.

The image in the upper left quadrant of Figure 3 is a fairly clean image. There are no astronomical features in the sky in this image, and on the horizon there are features from cities to the southeast and southwest. The light pollution in the upper right quadrant image appears far different; the sources to the southeast have disappeared, and the city to the southwest is apparently far larger. In reality, there are low lying clouds on the horizon in both directions; these have blocked the one city out completely. The city to the southwest is Tocopilla; it has fog on many occasions, which is thin enough to scatter light very well as in this image. The lower left image appears to show the lights from Tocopilla extending far into the sky; in this case that is the galaxy intersecting with the lights from Tocopilla on the horizon. Note also the difference in the lights to the southeast. In the final image, there are clouds on the horizon again, and more features on the sky; those features are due to the ASCA dome requiring a cleaning.

The difference between just this set of images, from a single site, shows the necessity of examining as many nights as possible from each of the sites. There are changes due to instrumentation, light sources appearing or disappearing over time, patches of clouds near light sources at times, and the appearance of the galaxy in the data. It is critically important to examine each light pollution analysis image, and raw data if necessary, to conclude what the different features are in the images, and then combine the analysis of several images to determine what the effects of light pollution at that site are.

\section{DATA ANALYSIS AND RESULTS}

There are five sites in the TMT site testing program. Each site has an ASCA as part of the site testing equipment suite. All five sites are very dark on average, with the only light pollution coming from nearby communities or work sites. For light pollution to be an issue for TMT, it must extend above the $65^{\circ}$ zenith angle that will be the lower limit for TMT observations.

Below is a discussion of the effects of light pollution at each of the sites. 

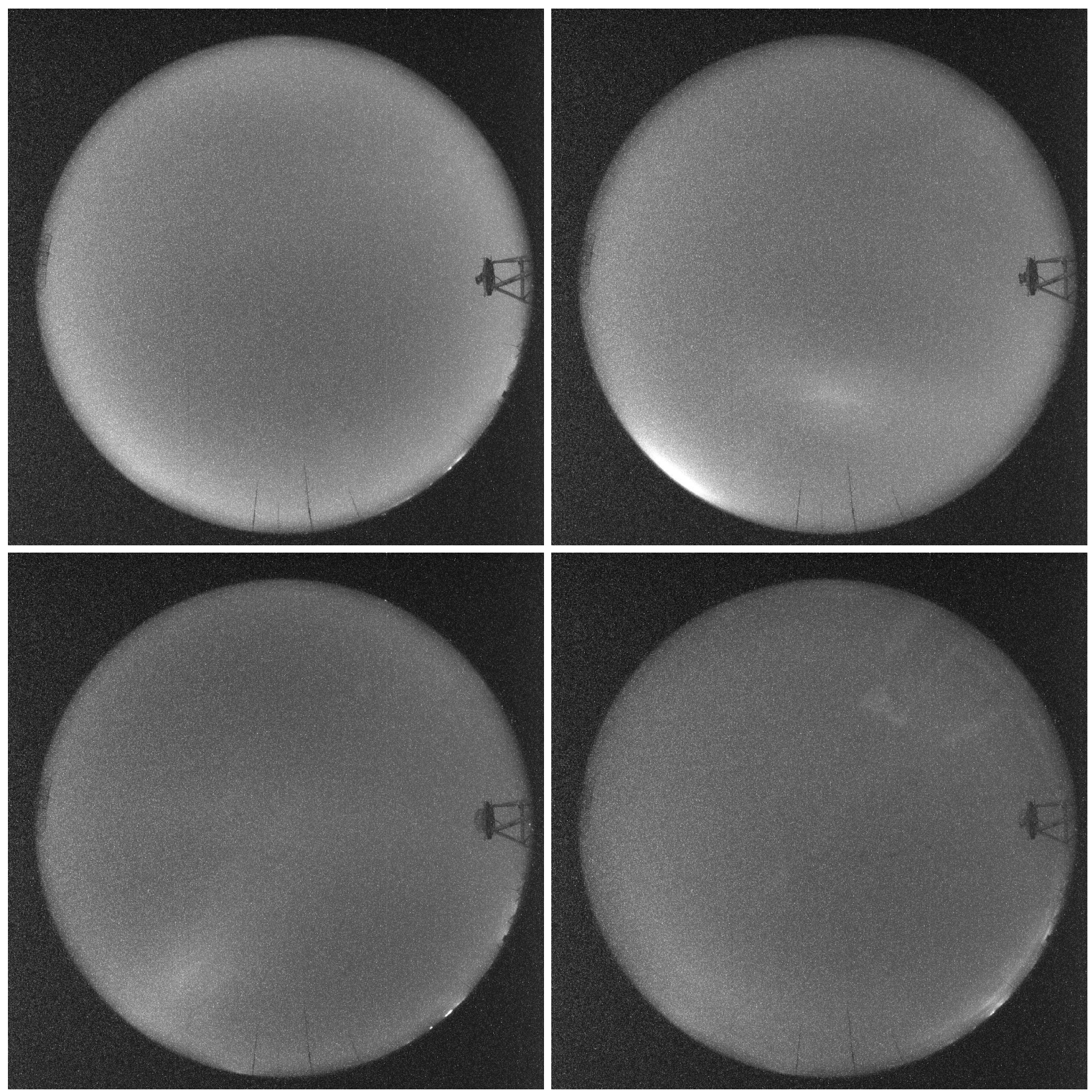

Figure 3. A set of light pollution analysis images from Cerro Tolar, showing the various features that can appear in the data.

\subsection{Cerro Tolar, Chile}

Cerro Tolar is located in the northern Atacama Desert in Chile, in a mountain range that runs along the coast to the north of Tocopilla. Figure 3 is a set of reduced ASCA images from Cerro Tolar. The only large scale light pollution feature are the lights of Tocopilla to the southwest when there are low clouds or fog in that area. The other set of lights almost directly to the southeast are from the lights of Maria Elena. There are also some lights further east that likely come from the city of Calama or possibly the Chuquicamata mine.

The light pollution analysis image with the fog over Tocopilla is likely the worst possible light pollution at Cerro Tolar; it also occurs at the worst time for TMT operations, with a clear sky. But, even under this scenario, 
the light scattered into the sky still stays near the horizon. Cerro Tolar is only $18 \mathrm{~km}$ from Tocopilla, but the bluffs above Tocopilla are $1000 \mathrm{~m}$ high and block most of the light produced in town. It is very unlikely that the light pollution from Tocopilla extends into the TMT beam.

\subsection{Cerro Armazones, Chile}

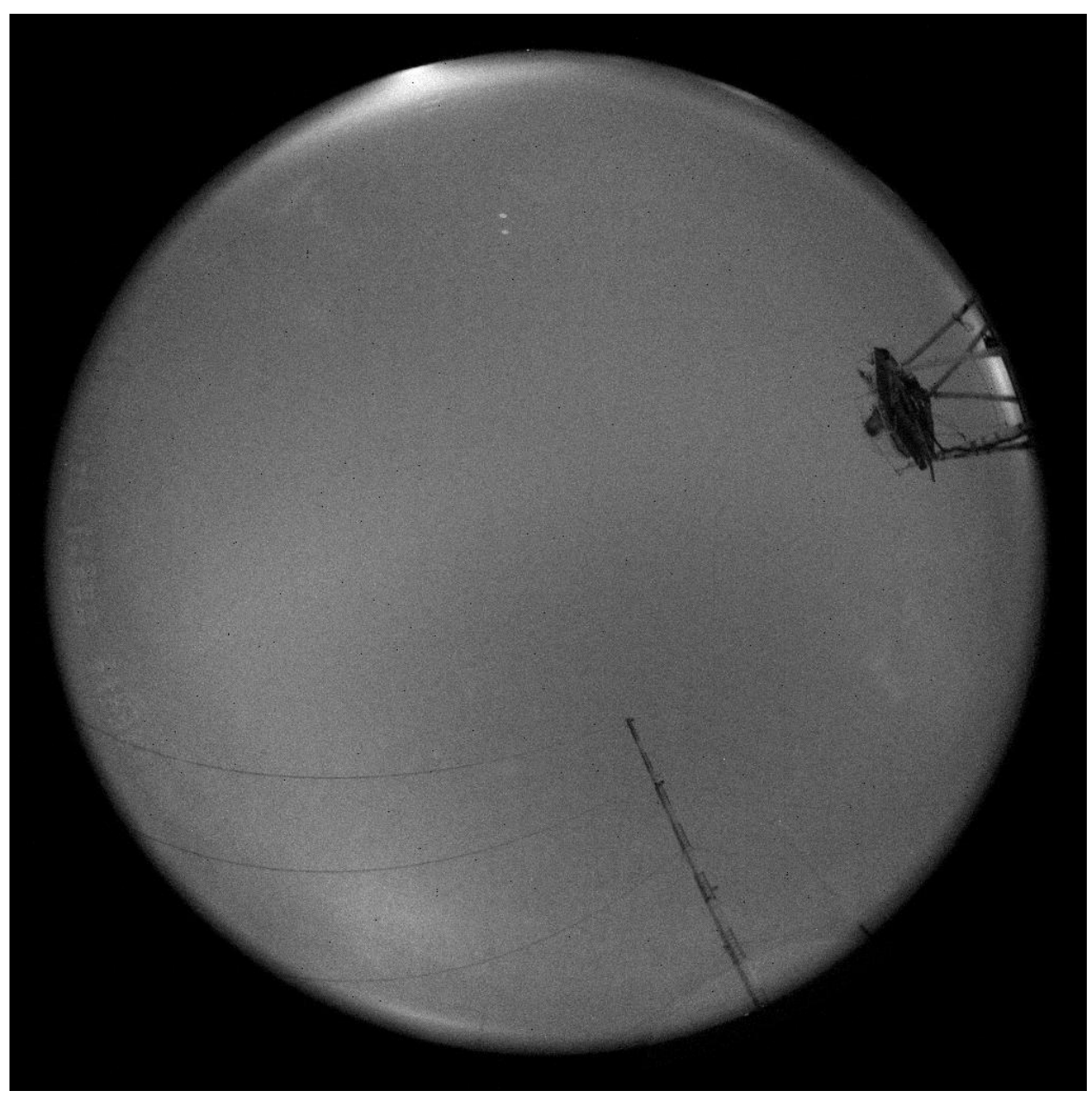

Figure 4. Light pollution at Cerro Armazones.

Figure 4 is once again Cerro Armazones, but a different night from the ones in Figures 1 and 2. As with one of the data images from Cerro Tolar, this night was clear above the site, but there were low lying clouds or fog above Antofagasta that scattered more light than usual. This night was the brightest of the light pollution images observed. Antofagasta, at a distance of $110 \mathrm{~km}$, clearly appears in the northwest quadrant of the image, with an extended area of light pollution. Another source of light is in the southwest quadrant; these are most likely the towns of Paposo and Taltal. The light source behind the base of the telescope tower is Mina Escondida (which is about $120 \mathrm{~km}$ away).

Even on this, the most light polluted data found for Armazones, the extent of the light pollution remains low on the horizon. There should be no effect on TMT from light pollution at this site. There is, however, some prospecting for mines happening around this region; a mine placed near Cerro Armazones could have a large impact on the light pollution environment. 


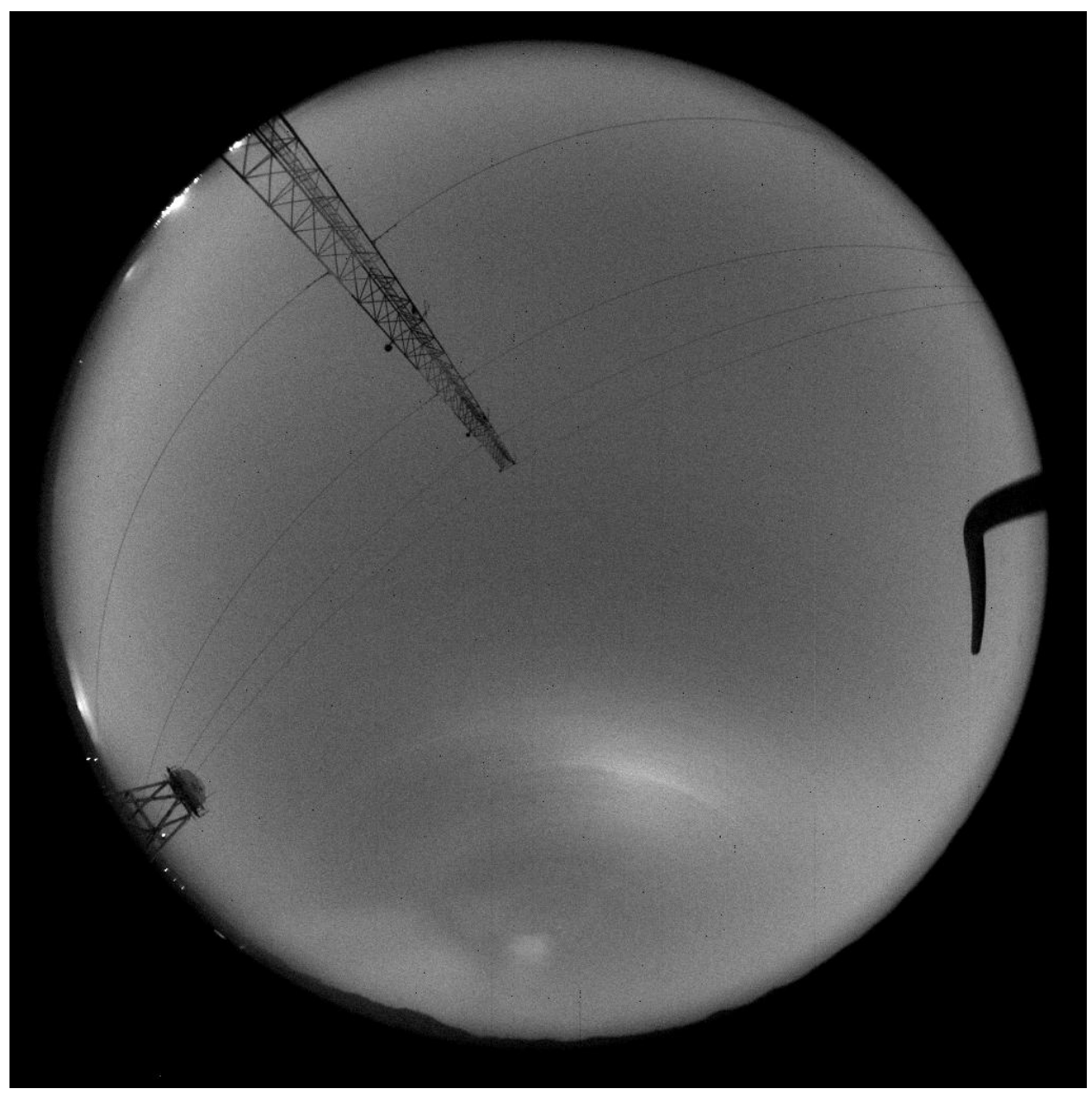

Figure 5. The light pollution image for Cerro Tolonchar.

\subsection{Cerro Tolonchar, Chile}

Cerro Tolonchar is the highest $(4480 \mathrm{~m})$ and most remote of all TMT candidate sites. The closest settlement is Socaire (300 inhabitants) $38 \mathrm{~km}$ to the north, with the closest towns being Toconao (550 people) at $80 \mathrm{~km}$, San Pedro de Atacama (an eco-tourism town of 1,500 people) at $115 \mathrm{~km}$ and Calama, the next large city with a commercial airport (120,000 people), at $190 \mathrm{~km}$.

It is also just southeast of the Salar de Atacama, in an area with a lot of mining activity. Those are the small lights around the site testing telescope (to the southwest) tower in Figure 5, aside from the one on the tower platform (which is a webcam light). To the west southwest, there is a large light source; this is again Mina Escondida, seen from a different angle than Cerro Armazones. The three light sources near the base of the tower in the northwest are the Chuquicamata mine, Calama and San Pedro de Atacama. The rest of the region around Cerro Tolonchar is sparsely populated, with the two closest communities (Socaire and Peine) not evident in the light pollution image. The galaxy is the source of the broad swath of light in the sky, and scattered light from dust or dirt on the ASCA enclosure is the cause of the artifacts near the bottom of the image. The eastern horizon is clear of any light sources; with the Andes mountains in this area (25-100 km away) that is unlikely to change.

All light sources are near the horizon, and the site is a dark site, so there should be no light effects for TMT in the future at this site, barring a explosion of mining in the area. 


\subsection{San Pedro Mártir, Mexico}

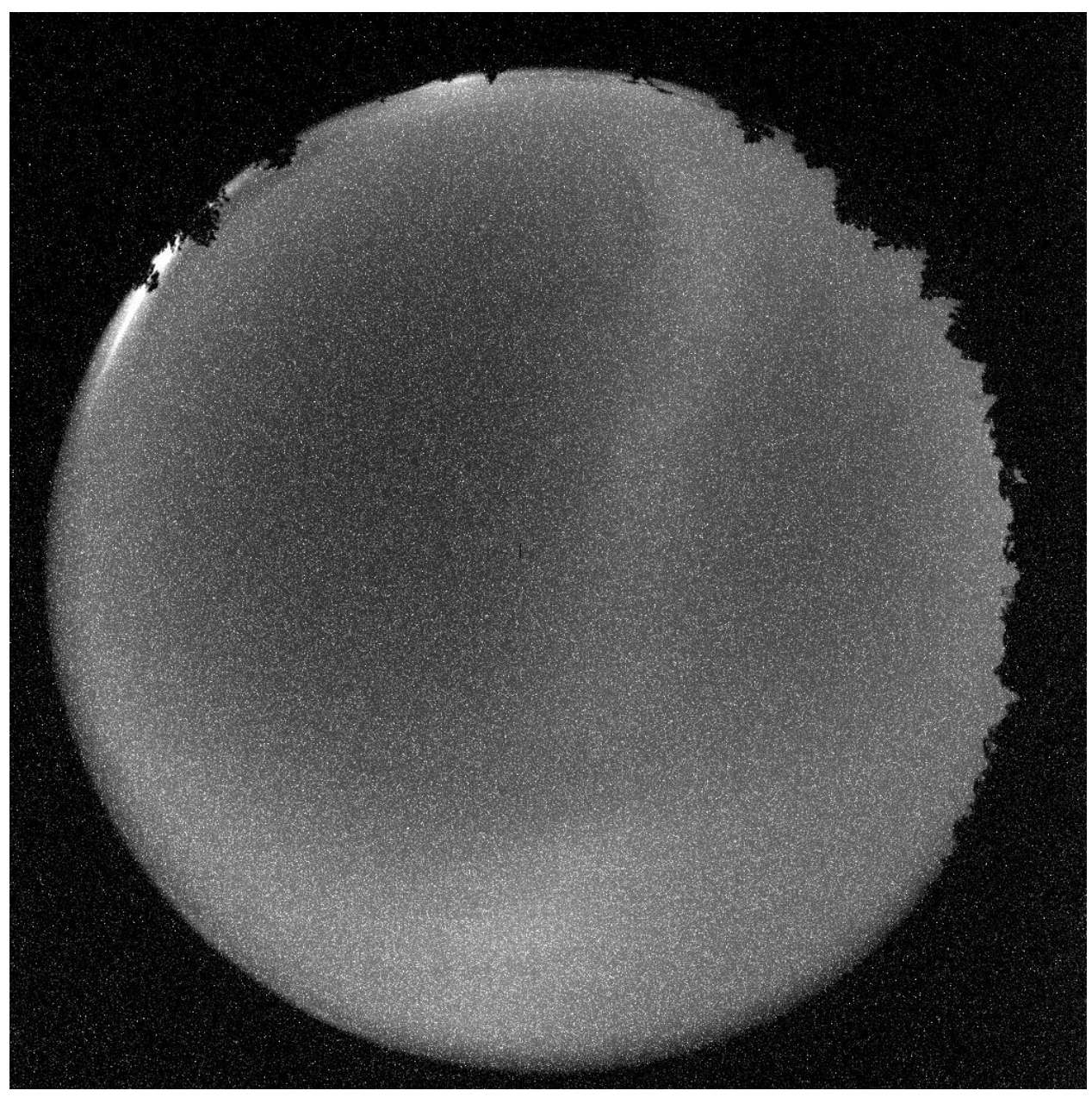

Figure 6. The light pollution image for San Pedro Martir in Mexico.

San Pedro Mártir (SPM) is located in northern Baja California, Mexico, inside a national park and is the site of the Observatorio Astronómico Nacional de San Pedro Mártir. It is a medium-elevation site (2830 m), 65 $\mathrm{km}$ from the Pacic coast in the west and $55 \mathrm{~km}$ from the Sea of Cortez (Gulf of California) to the east. The closest large town is Ensenada (300,000 inhabitants) at $140 \mathrm{~km}$. Tijuana (at $220 \mathrm{~km})$, Mexicali $(220 \mathrm{~km})$, and San Diego $(250 \mathrm{~km})$ are large cities in the vicinity.

Figure 6 shows the effects of light pollution in the area; this image has not been dark current corrected because there were no dark current images taken at that time. Overall, SPM is a dark site, and there are no signs of light pollution from the small settlements in the area (a few scattered communities to the west mainly). In fact, the only large sources of light pollution in the image come from the large towns that are hundreds of kilometers away. The largest source is Ensenada to the west northwest, with Tijuana/San Diego to the northwest, and Mexicali to the north.

There is a limited power grid in the area of SPM, which limits the growth of light pollution in the area. It is possible that increasing the power to the area could be a factor in the future, but economic growth in the area would have to increase vastly for this to become an issue. The large light sources are far enough away that they remain low on the horizon. None of these should be a factor for TMT in the future. 


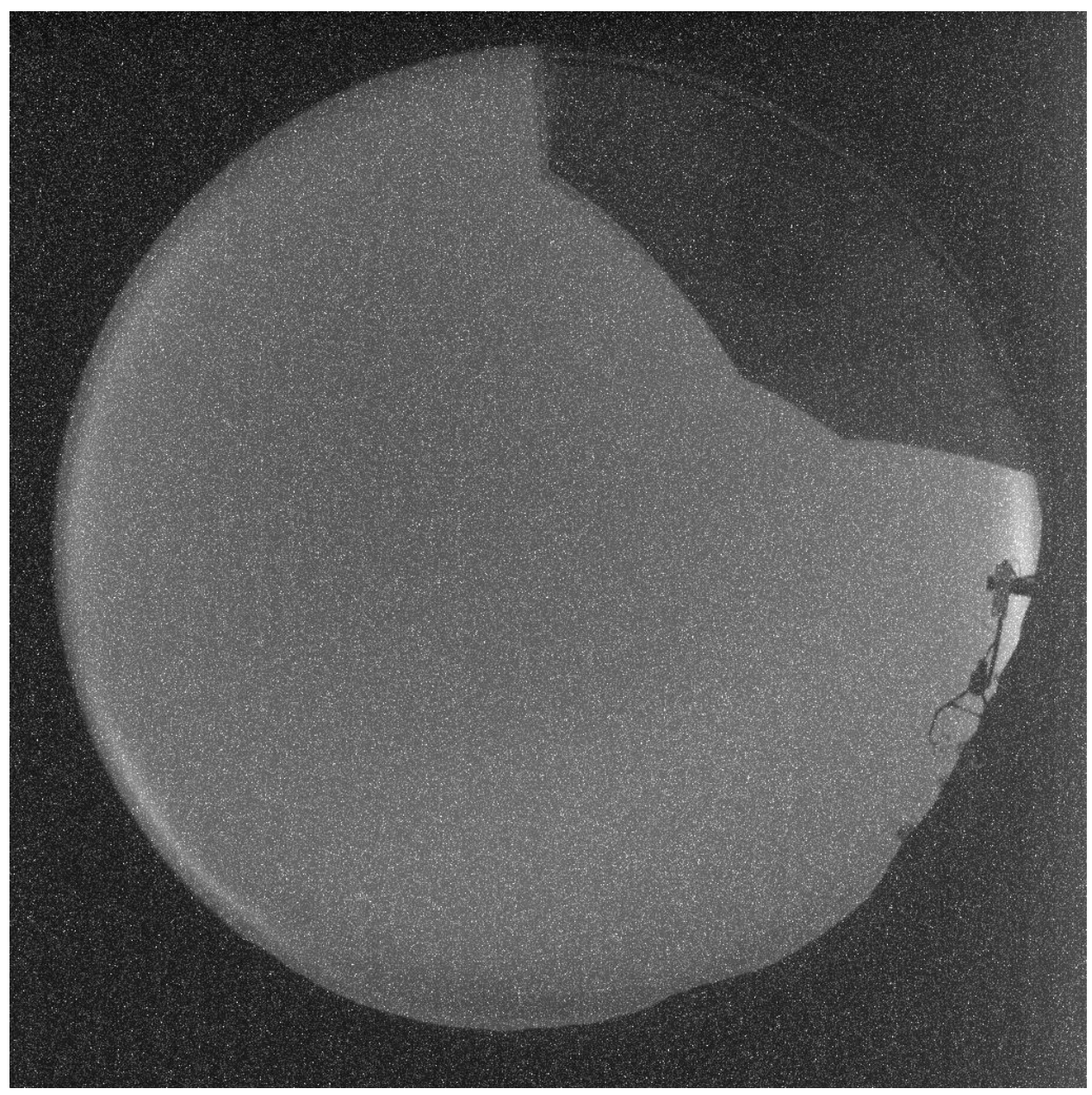

Figure 7. The light pollution image for Mauna Kea at $13 \mathrm{~N}$ in Hawai'i.

\subsection{Mauna Kea, 13 North, Hawai'i}

The TMT site testing equipment setup on Mauna Kea is not situated on the summit ridge. Instead, it is at the 13 North $(13 \mathrm{~N})$ site, which was marked out as the site for further development of a large telescope on Mauna Kea. Figure 6 shows the effects of light pollution in the area; this image has not been dark current corrected. The CCD used in the ASCA system at $13 \mathrm{~N}$ has sensitivity problems compared to the other systems, but the median processing of the images mitigates that somewhat; the magnitude of the measured light pollution might be lessened slightly as a result. ASCA is also mounted on the telescope tower, which blocks a portion of the sky.

Hilo (30 km away) is in this part of the sky; the lights from it are in the east. Hilo is south of east from the site, so the center of town (and the expected brightest source, though there is no line of sight from 13N) is in the image. There is also a band of lights from the southwest to northwest. The larger area to the southwest is Kona $(45 \mathrm{~km})$, while the fainter sources are other small towns or resorts on the island.

None of the light pollution sources are a concern now for TMT; they do not extend far enough into the sky. Growth is slowly occurring in the area around Mauna Kea, but with astronomy as an important focus for the island there is ample and continuing discussion of the light pollution problem. ${ }^{19}$

\subsection{Future analysis}

To this point, the light pollution analysis has concentrated on whether the sites show any effects from light pollution that would be an issue for TMT. All of the sites are dark sites, so the only concern is the pollution 
from cities. In the case of all of the sites, the measured light pollution is below the $65^{\circ}$ zenith distance limit for TMT.

Further analysis to quantify the result is underway. The difficulty in quantifying the result is that the ASCA units are not the same, and have not been cross calibrated against each other. Photometric correction for each image (through the use of known bright stars) is the only way to find comparable values between sites; that is not possible with the current analysis method. There is also the possibility of measuring the variation in the light pollution as a function of time; in the case of Cerro Tolonchar, this is especially interesting as light pollution controls were instituted in Chile during the time ASCA was in operation at the site.

\section{CONCLUSION}

A project was undertaken to determine if light pollution is an important factor for the site selection decision for the TMT project. A method was developed that used the ASCA instrument to measure light pollution, using a $10 \%$ median image to separate light pollution from astronomical effects in the ASCA images. An analysis of these light pollution data images demonstrates that all of the TMT sites are dark sites, and that each site shows some level of light pollution in the area. However, the light pollution in the area of the sites is below the zenith angle limit of $65^{\circ}$ for TMT, which was the important question to answer for TMT site selection to proceed.

Further analysis of the data is planned to quantify this result more fully, as well as to examine whether there are any temporal variations of the light pollution in the areas around the TMT testing sites.

\section{ACKNOWLEDGMENTS}

The authors gratefully acknowledge the support of the TMT partner institutions. They are the Association of Canadian Universities for Research in Astronomy (ACURA), the California Institute of Technology and the University of California. This work was supported as well by the Gordon and Betty Moore Foundation, the Canada Foundation for Innovation, the Ontario Ministry of Research and Innovation, the National Research Council of Canada, the Natural Sciences and Engineering Research Council of Canada, the British Columbia Knowledge Development Fund, the Association of Universities for Research in Astronomy (AURA) and the U.S. National Science Foundation.

\section{REFERENCES}

[1] Garstang, R. H., "Mount Wilson Observatory: the sad story of light pollution," The Observatory 124, 14-21 (Feb. 2004).

[2] Walker, M. F., "Light Pollution in California and Arizona," PASP 85, 508-519 (Oct. 1973).

[3] Riegel, K. W., "Light pollution. Outdoor lighting is a growing threat to astronomy.," Science 179, 1285-1291 (Mar. 1973).

[4] Upgren, A. R., Armandroff, T. E., and Dawson, D. W., "Results of a Pilot Program to Measure Light Pollution," in [Bulletin of the American Astronomical Society], Bulletin of the American Astronomical Society 13, 813 (Mar. 1981).

[5] Massey, P., Gronwall, C., and Pilachowski, C. A., "The spectrum of the Kitt Peak night sky," PASP 102, 1046-1051 (Aug. 1990).

[6] Tritton, K. P., "Astronomical requirements for limiting lighting pollution," The Observatory 117, 10-13 (Feb. 1997).

[7] Percy, J. R., "Light Pollution: Education of Students, Teachers and the Public," in [Preserving the Astronomical Sky], Cohen, R. J. and Sullivan, W. T., eds., IAU Symposium 196, 353 (2001).

[8] Murdin, P., [International Dark-Sky Association], Encyclopedia of Astronomy and Astrophysics (Nov. 2000).

[9] Schwarz, H. E., ed., [Light Pollution: The Global View], Astrophysics and Space Science Library 284 (Apr. 2003).

[10] Isobe, S., Hamamura, S., and Elvidge, C. D., "Educating the Public about Light Pollution," in [Preserving the Astronomical Sky], Cohen, R. J. and Sullivan, W. T., eds., IAU Symposium 196, 363 (2001). 
[11] Smith, M. G., "Protecting the Local Dark-Sky Areas around the International Observatories in Chile.," in [Bulletin of the American Astronomical Society], Bulletin of the American Astronomical Society 33, 1470 (Dec. 2001).

[12] Smith, M. G., Sanhueza, P., Norman, D., Schwarz, H., and Orellana, D., "Protection of Existing and Potential Astronomical Sites in Chile - an Update.," in [Bulletin of the American Astronomical Society], Bulletin of the American Astronomical Society 34, 1198 (Dec. 2002).

[13] Wace, M., "Light Pollution Reduction in Tucson Aids Kitt-Peak Astronomers," Journal of the British Astronomical Association 98, 133 (Apr. 1988).

[14] Semler, D. R., Schwarz, H., Smith, M., and Suntzeff, N., "15-year Sky Background Trends at CTIO," in [Bulletin of the American Astronomical Society], Bulletin of the American Astronomical Society 37, 1210 (Dec. 2005).

[15] Smith, M. G., Sanhueza, P., Schwarz, H. E., and Walker, A. R., "Is Chile Complying with its Light Pollution Regulations? A Status Report," in [Bulletin of the American Astronomical Society], Bulletin of the American Astronomical Society 37, 1211 (Dec. 2005).

[16] Walker, D. E., Schwarz, H. E., and Bustos, E., "Monitoring the night sky with the Cerro Tololo All-Sky camera for the TMT and LSST projects," in [Ground-based and Airborne Telescopes. Edited by Stepp, Larry M.. Proceedings of the SPIE, Volume 6267, pp. 62672O (2006).], Presented at the Society of Photo-Optical Instrumentation Engineers (SPIE) Conference 6267 (July 2006).

[17] Stealth Computer Corporation Website, http://www.stealthcomputer.com/home.htm.

[18] Skidmore, W., Schöck, M., Magnier, E., Walker, D., Riddle, Reed Els, S., Bustos, E., Seguel, J., and Paul, G., "Using All Sky Cameras to determine cloud statistics for the Thirty Meter Telescope candidate sites," in [Ground-based and Airborne Telescopes. Edited by Stepp, Larry M.. Proceedings of the SPIE, Volume 7012, paper 7012-76 (2008).], Presented at the Society of Photo-Optical Instrumentation Engineers (SPIE) Conference 7012 (June 2008).

[19] Wainscoat, R. J., "Protection of Mauna Kea and Haleakala Observatories from Light Pollution (with color plate)," in [Light Pollution: The Global View], Schwarz, H. E., ed., Astrophysics and Space Science Library 284, 111 (Apr. 2003). 